\title{
Impact of Identification and Treatment of Depression in Heart Transplant Patients
}

\author{
Ike Okwuosa, ${ }^{1}$ Dara Pumphrey, ${ }^{2}$ Jyothy Puthumana, ${ }^{3}$ \\ Rachel-Maria Brown, ${ }^{1}$ and William Cotts ${ }^{3}$ \\ ${ }^{1}$ Northwestern Memorial Hospital, Feinberg School of Medicine Department of Medicine, 251 E Huron, \\ Northwestern University, Chicago, IL 60616, USA \\ ${ }^{2}$ Feinberg School of Medicine Department of Psychiatry, Northwestern University, Chicago, IL 60616, USA \\ ${ }^{3}$ Feinberg School of Medicine Department of Medicine, Division of Cardiology, Northwestern University, Chicago, IL 60616, USA
}

Correspondence should be addressed to Ike Okwuosa; isokwuosa@gmail.com

Received 28 May 2014; Revised 23 August 2014; Accepted 29 August 2014; Published 14 September 2014

Academic Editor: Kate Scott

Copyright (C) 2014 Ike Okwuosa et al. This is an open access article distributed under the Creative Commons Attribution License, which permits unrestricted use, distribution, and reproduction in any medium, provided the original work is properly cited.

Background. The effects of clinical depression after orthotopic heart transplantation (OHT) are relatively unknown. The purpose of this study was to evaluate the impact of depression on outcomes after OHT. Methods. We performed a single center retrospective review of 102 consecutive patients who underwent OHT at Northwestern Memorial Hospital from June 2005 to October 2009. The diagnosis of depression was obtained from attending physician documentation. The primary endpoints were all-cause mortality (ACM), hospitalizations, and rejection. Results. Of 102 OHT patients, 26 (26\%) had depression. Depressed patients were similar in age to nondepressed patients (57.6 years versus 56.9, $P=0.79$ ). There was no statistical difference in survival between groups at 5 years after OHT $(P=0.94)$. All-cause hospitalizations were higher in depressed versus nondepressed patients (4.3 versus 2.6 hospitalizations $P=0.05$ ). There were no significant differences in hospitalizations between the two groups for the following complications: cardiac (heart failure, edema, arrhythmias, and acute rejection) and infections. There was no significant difference in episodes of $2 \mathrm{R}$ and $3 \mathrm{R}$ rejection. Conclusion. Early identification and treatment of depression in OHT patients result in outcomes similar to nondepressed patients.

\section{Introduction}

Heart failure is associated with significant resource utilization, accounting for nearly 1 million hospital admissions annually, placing a significant financial burden on the healthcare system [1]. In $2010 \$ 24$ billion were dedicated to care for heart failure patient; by 2030 that cost is projected to total $\$ 70$ billion [2]. Similar to other chronic diseases such as diabetes mellitus and chronic obstructive pulmonary disease, depression affects an estimated $20 \%$ of heart failure patients [3-5], compared to the estimated $6.6 \%$ amongst the general population [6].

OHT is definitive and the best treatment option available for patients with end stage heart failure. Following heart transplantation patients report improved functional capacity and overall quality of life; however this varies based on severity of illness before transplant [7, 8]. A significant portion of patients after OHT experience depression (estimated prevalence of $20-30 \%)[9,10]$.

Independently, depression has been associated with increased resource utilization. It is estimated that depression is responsible for $\$ 34$ billion in direct and indirect costs annually [11]. In patients with chronic diseases, depression has been associated with increased morbidity and mortality, when compared to nondepressed patients [12,13]. Depression has also been associated with medication nonadherence and increased hospitalizations. In a patient population with $\mathrm{OHT}$, this could have serious adverse outcomes and represents a serious challenge in posttransplant management.

Studies have shown that early identification and initiation of treatment for depression are associated with improved outcomes [14]. Antidepressant medication represents the 
best established treatment for patients with a diagnosis of depression [15]. So it can be implied that early identification and treatment of depression in OHT patients should lead to favorable outcomes.

Current studies evaluating depression and its effects in the OHT patient population have focused primarily on quality of life [16-20]. To our knowledge, no study to date has evaluated the impact of early identification and treatment of depression on survival, rehospitalizations, and organ rejection.

\section{Methods}

2.1. Study Design. The study was reviewed and approved by the internal review board. This study was performed as a single center retrospective electronic medical record (EMR) review of consecutive patients who underwent OHT at Northwestern Memorial Hospital from June 2005 through October 2009. The Diagnostics and Statistical Manual Fourth Edition-Text Revision (DSM-IV-TR) defines major depressive disorder as "a mood disorder having a clinical course involving one or more episodes of serious psychological depression that last two or more weeks each, do not have intervening episodes of mania or hypomania, and are characterized by a loss of interest or pleasure in almost all activities and by some or all of disturbances of appetite, sleep, or psychomotor functioning, a decrease in energy, difficulties in thinking or making decisions, loss of self-esteem or feelings of guilt, and suicidal thoughts or attempts." For the purpose of our study we selected patients who were diagnosed as having depression as verified by attending physician documentation in the medical record. We did not utilize the DSM-IV-TR criteria for major depressive disorder. Using stringent criteria as this would limit our study as this information was not readily available in our medical records. This would have also resulted in a less inclusive study that would ultimately be less generalizable, as many individuals with medical illness suffer from some depressive symptoms and do not meet criteria for major depressive disorder. Making the diagnosis of major depressive disorder can also be challenging in individuals with chronic illnesses as some of the symptoms of major depressive disorder (e.g., decreased appetite and decreased energy) overlap with symptoms of chronic illnesses themselves. All patients underwent pretransplantation evaluation by the Cardiovascular Behavioral Medicine team, and depressed patients were further evaluated by the inpatient Psychiatric Liaison service. The retrospective nature of this study limited the availability of severity of depression. Inpatient and outpatient medical records were reviewed for antidepressant medications. Baseline characteristics including the prior use of mechanical circulatory assist devices were obtained. Complete follow-up information including total hospitalizations subcategorized into cardiac and infectious hospitalizations was obtained. Cardiac hospitalizations included hospitalization for arrhythmias, acute coronary syndrome, chest pain, shortness of breath, cardiac rejection, and decompensated heart failure. Infectious hospitalizations were defined as any hospitalization for a documented fever, bacteremia, viremia, fungemia, and pneumonia. Pathologist confirmed records were reviewed for the presence of International Society for
Heart and Lung Transplant (ISHLT) defined rejection [21] (please see Table 3).

Primary endpoints were ACM, ISHLT defined rejection of $2 \mathrm{R}$ and greater, and hospitalizations for infectious or cardiac etiologies. Secondary endpoints were time to initial hospitalization and hospitalizations for any cause and average serum levels of tacrolimus at 6 months after transplant.

2.2. Data Analysis. Baseline characteristics between depressed and nondepressed patients were compared using the $\chi^{2}$ test for categorical variables. Overall survival was assessed using the Kaplan-Meier method. Time to first hospitalization was assessed utilizing a modified Kaplan-Meir method. The curves were compared using a log-rank test.

Data were analyzed using SAS statistical software version 9.1 (SAS Institute Inc.). All statistical tests were 2-sided, and a probability value of less than 0.05 was used to define statistical significance.

\section{Results}

In the study period, a total of 102 patients underwent OHT. 26 patients $(26 \%)$ were diagnosed with depression (80\% preOHT and $20 \%$ post-OHT). Of the patients with depression $92 \%$ were on an antidepressant medication (79\% selective serotonin reuptake inhibitor, SSRI; 13\% Other antidepressants; $8 \%$ serotonin norepinephrine reuptake inhibitor, SNRI) and the remaining $8 \%$ received psychotherapy weekly. Baseline characteristics were similar (Table 1). The transplant group was predominately Caucasian ( $81 \%$ versus $75 \%, P=$ $0.53)$ and male $(65 \%$ versus $76 \%, P=0.23)$. Mean age was similar between the patients with depression versus nondepressed patients (57.6 versus 56.9, $P=0.79$ ). The etiology of cardiomyopathy and LVAD use prior to OHT was similar between the two patient groups ( $46 \%$ versus $45 \%$ $P=0.92$ ) prior to $\mathrm{OHT}$.

\section{Survival}

There was no overall statistical difference in survival over a 5 -year period (Figure 1) between the two groups. There is a slight trend favoring the depressed cohort.

\section{Rejection}

As indicated in Table 2, rejection episodes and severity of rejection (ISHLT grade $2 \mathrm{R}$ and greater) were not statistically different between depressed and nondepressed patients. Serum tacrolimus levels were not statistically different 6 months after OHT.

\section{Hospitalizations}

Time to first hospitalization is illustrated in Figure 2. Median time to first hospitalization was identical in both groups at 88 days. All-cause hospitalizations were higher in the depressed group versus the nondepressed group (4.3 versus 2.6 hospitalizations per patient, $P=0.05)$. There was no statistical significance between the two groups for cardiac (1.19 
TABLE 1: Baseline characteristics.

\begin{tabular}{lccc}
\hline Characteristics & $\begin{array}{c}\text { Depressed } \\
(N=26)\end{array}$ & $\begin{array}{c}\text { Nondepressed } \\
(N=75)\end{array}$ & $P$ value \\
\hline Age & $57.6 \pm 12.3$ & $56.8 \pm 14$ & .79 \\
Gender & Male 65\% (17) & Male 76\% (58) & .0027 \\
Remale 35\% (9) & Female 23\% (17) & .0027 \\
Caucasian & $81 \%(21)$ & $75 \%(56)$ & .53 \\
African American & $15 \%(4)$ & $15 \%(11)$ & 1.00 \\
Hispanic & $4 \%(1)$ & $5 \%(4)$ & .8 \\
Asian & $0 \%$ & $5 \%(4)$ & .24 \\
Ischemic cold time & 183 & 196 & .14 \\
LVAD & $46 \%$ & $45 \%$ & .92 \\
Etiology & & & \\
ICM & $38 \%$ & $39 \%$ & .92 \\
DCM & $42 \%$ & $37 \%$ & .65 \\
Valv & $4 \%$ & $5 \%$ & .83 \\
Myo & $0 \%$ & $3 \%$ & .37 \\
Chemo & $12 \%$ & $11 \%$ & .88 \\
Cong & $4 \%$ & $1 \%$ & .32 \\
Fam & $0 \%$ & $3 \%$ & .37 \\
TCAD & $0 \%$ & $1 \%$ & .60 \\
\hline
\end{tabular}

LVAD = left ventricular assist device, ICM = ischemic cardiomyopathy, DCM = dilated cardiomyopathy, Valv = valvular cardiomyopathy, Myo = myocarditis Cong = congenital cardiomyopathy, Fam = familial cardiomyopathy, Chemo $=$ chemotherapy induced cardiomyopathy, and TCAD $=$ transplant coronary artery disease.

TABLE 2: Rejections episodes by depression status, $N(\%)$.

\begin{tabular}{cccc}
\hline Rejection & Depression & No depression & $P$ value \\
\hline 0R & $26(100.0 \%)$ & $74(100.0 \%)$ & $\mathrm{n} / \mathrm{a}$ \\
1R & $25(96.2 \%)$ & $69(93.2 \%)$ & 1.000 \\
2R & $6(23.1 \%)$ & $22(29.7 \%)$ & 0.516 \\
1 & $2(7.7 \%)$ & $18(24.0 \%)$ & \\
2 & $2(7.7 \%)$ & $3(4.0 \%)$ & \\
3 & $0(0.0 \%)$ & $1(1.3 \%)$ & \\
4 & $1(3.9 \%)$ & $0(0.0 \%)$ & \\
5 & $1(3.9 \%)$ & $0(0.0 \%)$ & \\
$3 \mathrm{R}$ & $1(3.9 \%)$ & $4(5.4 \%)$ & 1.000 \\
1 & $1(3.9 \%)$ & $3(4.0 \%)$ & \\
2 & $0(0.0 \%)$ & $1(1.3 \%)$ & \\
$4 \mathrm{R}$ & $0(0.0 \%)$ & $0(0.0 \%)$ & $\mathrm{n} / \mathrm{a}$ \\
\hline
\end{tabular}

versus $0.63, P=0.11)$ or infectious ( 0.8 versus $0.96, P=0.58$ ) hospitalizations (Figure 3).

\section{Discussion}

In our study of 102 consecutive OHT patients we found that the outcomes between patients with and without clinical depression did not differ in survival, cardiac or infectious hospitalizations, and organ rejection. Further the serum levels of immunosuppressant were similar between the groups
TABLE 3: International Heart and Lung Transplant Society acute rejection criteria.

\begin{tabular}{|c|c|}
\hline Grade & Description \\
\hline OR & No evidence of acute cellular rejection \\
\hline $1 \mathrm{R}$ & $\begin{array}{l}\text { Mononuclear cells infiltration without or with only one } \\
\text { focus of myocyte damage }\end{array}$ \\
\hline $2 \mathrm{R}$ & $\begin{array}{l}\text { An infiltrate plus the presence of multifocal myocyte } \\
\text { damage }\end{array}$ \\
\hline $3 \mathrm{R}$ & $\begin{array}{l}\text { An infiltrate with diffuse myocyte damage and/or } \\
\text { associated edema, hemorrhage, or vasculitis }\end{array}$ \\
\hline
\end{tabular}

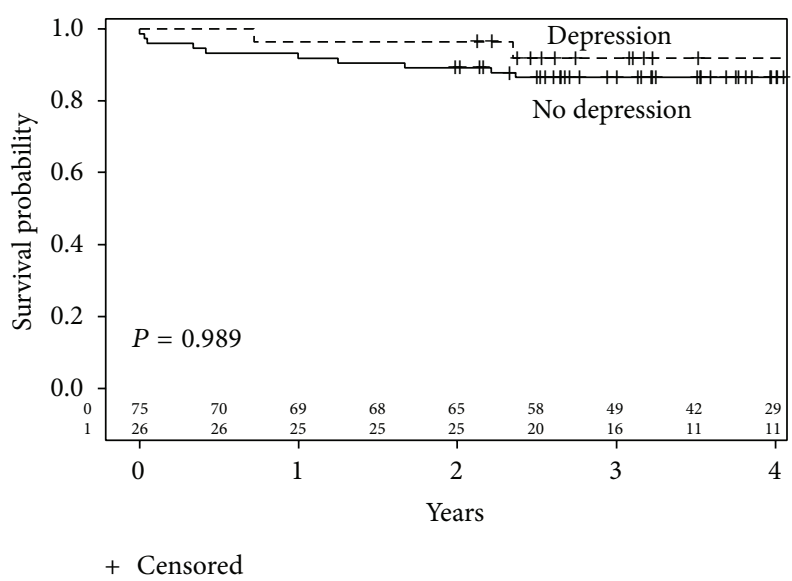

\begin{tabular}{|l|c|c|c|}
\hline Status & 1-year survival & 2-year survival & 3-year survival \\
\hline Depression & $96.2 \%$ & $96.2 \%$ & $92.0 \%$ \\
No depression & $92.0 \%$ & $89.3 \%$ & $86.5 \%$ \\
Total & $93.1 \%$ & $91.1 \%$ & $87.9 \%$ \\
\hline
\end{tabular}

Figure 1: Kaplan-Meier overall survival curves by depression status $(\log$-rank $P$ value $=0.989)$.

during the follow-up period. The prevalence of depression in this cohort was $26 \%$, with $92 \%$ of these patients receiving antidepressant medications. $80 \%$ of the depressed patients were diagnosed before OHT and the remaining $20 \%$ were diagnosed within 1 year after OHT.

Multiple studies have demonstrated an association between increased morbidity and mortality in depressed patients with concomitant cardiovascular disease compared to nondepressed patients [22-25]. Zipfel et al. in a study evaluating the effects of depression in the post-heart-transplant patient demonstrated that patients with high depression scores and an underlying etiology of ischemic cardiomyopathy were at increased risk of mortality after transplant [26]. Bush et al. underscore this concept but highlight that the presence of minimal symptoms of depression increases the risk of mortality [27]. Havik et al. demonstrated similar findings, with depression being an independent predictor of mortality in post-heart-transplant patients [28]. Studies have shown that depressed patients are more likely not to comply with prescribed medications, which would be devastating in a heart transplant patient [29-32]. Dew et al. identified specific risk factors that would predict poor post-heart-transplant outcomes in the depressed patient cohort, and they include 


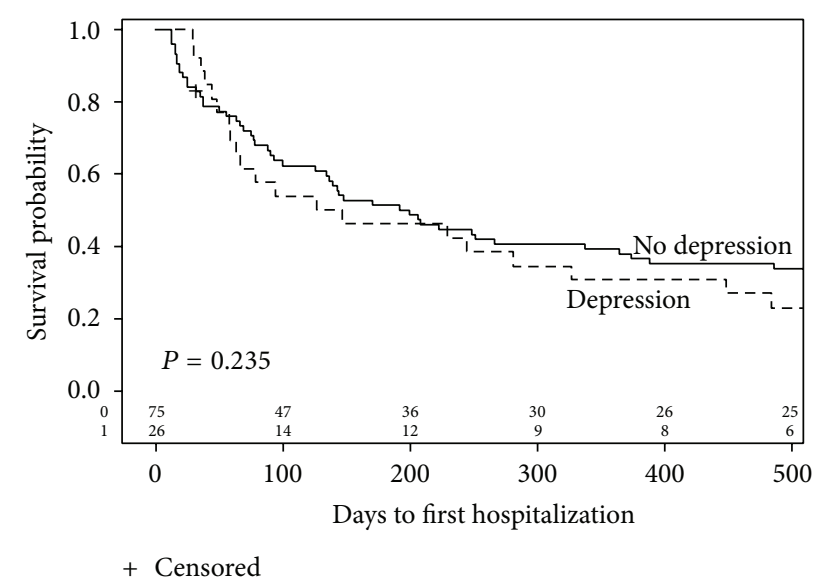

FIgURE 2: Kaplan-Meier curves for time-to-first hospitalization by depression status (log-rank $P$ value $=0.235$ ).



FIgURE 3: Post-heart-transplant hospitalizations. Hospitalization frequency between depressed and nondepressed patients. Depressed patients on average were hospitalized more frequently, $P=0.05$. No statistical difference for infectious $(P=0.58)$ and cardiac hospitalizations $(P=0.11)$.

poor social support, the use of avoidance coping strategies for health problems, and low self-esteem [33]. Dobbels et al. stress the importance of pretransplant screening, particularly focusing on self-reported nonadherence, lower social support, and education levels [34] to lower the risk of complications and organ rejection after OHT. Owen et al. in study assessing posttransplant complications followed 108 transplant recipients for approximately 3 years and identified previous suicide attempts, nonadherence to medical recommendations, and medications, alcohol, and substance abuse as risk factors for decreased survival. They further identified previous suicide attempt as an independent risk factor for posttransplant infections and speculated that early intervention may promote improved outcomes in this patient population [35].

Our results differ from the previous OHT studies in that our study population was initiated on antidepressant medications or was undergoing psychotherapy treatment either before transplant or within one year after transplant. Rogal et al. in a recent study looking at the impact of antidepressant use among patients identified with depression before liver transplant found similar findings that there was no difference in survival and organ rejection when compared to nondepressed patients [36]. Depression is one of the most common and debilitating illnesses that frequently goes undiagnosed and when diagnosed is frequently undertreated [37, 38]. Early identification and initiation of treatment of depression have been shown to improve outcomes as shown in a study by Berkman et al., where patients after myocardial infarction (MI) were initiated on treatment for depression. This study demonstrated a reduction in depressive symptoms and no difference in survival after MI between depressed and nondepressed patients $[38,39]$.

The ISHLT in the most recent cardiac transplant guidelines recommends routine screening for depressive symptoms [40]. Heart transplant patients are at increased risk of developing major depressive disorders because of the high incidence of depression after OHT, and regular screening and early intervention should be initiated in this patient population [41]. The Patient Health Questionnaire 9 (PHQ-9) is a self-administered diagnostic tool for common mental disorders. The PHQ-9 has been validated as a diagnostic test that can be administered in an office setting and has the advantages of objectively monitoring depressive severity and response to therapeutic management [42-44]. The PHQ-9 can be administered to patients over the phone and has been shown in a study to have similar sensitivity in identifying depression when compared to in-person screening [45].

Organ transplantation involves the allocations of scarce resources. In a large cost analysis study DiGiorgi et al. estimate the cost of initial hospitalization for OHT to be $\$ 124,830$ and $\$ 6,356$ for readmissions [46]. Shireman et al. estimated annual cost of graft maintenance to be $\$ 58,093$ [47]. Studies have shown that untreated depression is associated with increased healthcare utilization [48-50]. Hence, based on our findings early identification and treatment of depression in OHT patients provide comparable outcomes to OHT recipients without depression and possibly less resource utilization. Heart transplant and posttransplantation care has evolved over the past half century; Bruschi et al. in a study highlight a 25-year experience of the complexities of the management of posttransplant care and how careful patient selection, clinical follow-up, and multidisciplinary teamwork involving heart failure experts and other allied health professionals led to excellent long term results [51].

The retrospective nature of our study and the sample size may limit the generalizability of these findings. However, our OHT patients obtain all of their follow-up care at a single institution, and we feel that all outcomes are adequately captured from our chart review. All patients underwent clinical evaluation by the cardiac behavioral medicine service, and depressed patients were further evaluated by the inpatient psychiatric liaison service. The diagnosis of depression was based on documentation by the treating heart failure physician after clinical evaluation by cardiac behavioral medicine and psychiatric consultation. Since the diagnosis of depression was made by different consultant psychiatrists, the interobserver variability may be a limitation of our study; 
however, all these patients were evaluated by the same cardiac behavioral medicine team and hence we are confident in the diagnosis. The medications used for management of depression varied and were dependent on physician preference. Hence, there is a possibility that different medications may have varying effects in the OHT population. A prospective study using an algorithmic approach to drug selection for treating depression that do not have significant interactions with the transplant medications would overcome this limitation and is worthy of future research. The retrospective nature of this study is further limited by the possibility of the underdiagnosis of depression. It is possible that patients who developed depressive symptoms following OHT were not identified and thus have been categorized as nondepressed, further supporting a prospective study that frequently assesses depressive symptoms.

In conclusion, we have shown that early identification and treatment of depression in OHT patients are associated with outcomes similar to nondepressed patients and have the potential to result in decreased resource utilization. A larger study that looks at prospectively identifying and treating depression in OHT patients with long term follow-up for outcomes is warranted.

\section{Abbreviations \\ OHT: Orthotopic heart transplant \\ ACM: All-cause mortality \\ LVAD: Left ventricular assist device \\ EMR: Electronic medical record \\ IHLT: International Heart and Lung Transplant.}

\section{Conflict of Interests}

The authors declare that there is no conflict of interests regarding the publication of this paper.

\section{Acknowledgments}

The authors would like to thank Dr. Clyde Yancy from Northwestern University Feinberg School of Medicine Division of Cardiology, Dr. John Franklin from Northwestern University Feinberg School of Medicine Department of Psychiatry, and Dr. Ijeoma Chukwu from the Department of Psychiatry, University of California, Irvine, for assistance in this project.

\section{References}

[1] V. L. Roger, A. S. Go, D. M. Lloyd-Jones et al., "Heart disease and stroke statistics-2011 update," Circulation, vol. 12, pp. 3:e183:e209, 2011.

[2] P. A. Heidenreich, J. G. Trogdon, O. A. Khavjou et al., "Forecasting the future of cardiovascular disease in the United States," Circulation, vol. 123, no. 8, pp. 933-944, 2011.

[3] T. Rutledge, V. A. Reis, S. E. Linke, B. H. Greenberg, and P. J. Mills, "Depression in heart failure. a meta-analytic review of prevalence, intervention effects, and associations with clinical outcomes," Journal of the American College of Cardiology, vol. 48, no. 8, pp. 1527-1537, 2006.
[4] R. Norwood, "Prevalence and impact of depression in chronic obstructive pulmonary disease patients," Current Opinion in Pulmonary Medicine, vol. 12, no. 2, pp. 113-117, 2006.

[5] L. E. Egede, "Effect of comorbid chronic diseases on prevalence and odds of depression in adults with diabetes," Psychosomatic Medicine, vol. 67, no. 1, pp. 46-51, 2005.

[6] "Current depression among adults-United States, 2006 and 2008," The Journal of the American Medical Association, vol. 304, pp. 2233-2235, 2010.

[7] J. Butler, N. S. McCoin, I. D. Feurer et al., "Modeling the effects of functional performance and post-transplant comorbidities on health-related quality of life after heart transplantation," Journal of Heart and Lung Transplantation, vol. 22, no. 10, pp. 1149-1156, 2003.

[8] L. Petrucci, S. Ricotti, I. Michelini et al., "Return to work after thoracic organ transplantation in a clinically-stable population," European Journal of Heart Failure, vol. 9, no. 11, pp. 1112-1119, 2007.

[9] M. A. Dew, R. L. Kormos, A. F. DiMartini et al., "Prevalence and risk of depression and anxiety-related disorders during the first three years after heart transplantation," Psychosomatics, vol. 42, no. 4, pp. 300-313, 2001.

[10] F. Dobbels, S. De Geest, S. Martin, J. Van Cleemput, W. Droogne, and J. Vanhaecke, "Prevalence and correlates of depression symptoms at 10 years after heart transplantation: Continuous attention required," Transplant International, vol. 17, no. 8, pp. 424-431, 2004.

[11] W. F. Stewart, J. A. Ricci, E. Chee, S. R. Hahn, and D. Morganstein, "Cost of lost productive work time among US workers with depression," The Journal of the American Medical Association, vol. 289, no. 23, pp. 3135-3144, 2003.

[12] M. L. Chung, T. A. Lennie, R. L. Dekker, J. R. Wu, and D. K. Moser, "Depressive symptoms and poor social support have a synergistic effect on event-free survival in patients with heart failure," Heart and Lung, vol. 40, no. 6, pp. 492-501, 2011.

[13] J. Jünger, D. Schellberg, T. Müller-Tasch et al., "Depression increasingly predicts mortality in the course of congestive heart failure," European Journal of Heart Failure, vol. 7, no. 2, pp. 261267, 2005.

[14] D. J. Kupfer, E. Frank, and J. M. Perel, “The advantage of early treatment intervention in recurrent depression," Archives of General Psychiatry, vol. 46, no. 9, pp. 771-775, 1989.

[15] J. C. Fournier, R. J. DeRubeis, S. D. Hollon et al., "Antidepressant drug effects and depression severity: a patient-level metaanalysis," JAMA - Journal of the American Medical Association, vol. 303, no. 1, pp. 47-53, 2010.

[16] B. Campbell and G. Etringer, "Posttransplant quality of life issues: depression-related noncompliance in cardiac transplant patients," Transplantation Proceedings, vol. 31, no. 4, pp. 59S60S, 1999.

[17] D. C. Fisher, K. D. Lake, T. J. Reutzel, and R. W. Emery, “Changes in health-related quality of life and depression in heart transplant recipients," Journal of Heart and Lung Transplantation, vol. 14, no. 2, pp. 373-381, 1995.

[18] P. Fusar-Poli, V. Martinelli, C. Klersy et al., "Depression and quality of life in patients living 10 to 18 years beyond heart transplantation," Journal of Heart and Lung Transplantation, vol. 24, no. 12, pp. 2269-2278, 2005.

[19] E. W. Ruzyczka, I. Milaniak, P. Przybyłowski et al., "Depression and quality of life in terms of personal resources in heart transplant recipients," Transplantation Proceedings, vol. 43, no. 8, pp. 3076-3081, 2011. 
[20] H. Spaderna, D. Zahn, S. Schulze Schleithoff et al., "Depression and disease severity as correlates of everyday physical activity in heart transplant candidates," Transplant International, vol. 23, no. 8, pp. 813-822, 2010.

[21] M. Billingham and J. A. Kobashigawa, "The revised ISHLT heart biopsy grading scale," Journal of Heart and Lung Transplantation, vol. 24, no. 11, p. 1709, 2005.

[22] R. Schulz, S. R. Beach, D. G. Ives, L. M. Martire, A. A. Ariyo, and W. J. Kop, "Association between depression and mortality in older adults: the cardiovascular health study," Archives of Internal Medicine, vol. 160, no. 12, pp. 1761-1768, 2000.

[23] R. M. Carney and K. E. Freedland, "Depression, mortality, and medical morbidity in patients with coronary heart disease," Biological Psychiatry, vol. 54, no. 3, pp. 241-247, 2003.

[24] J. C. Barefoot and M. Schroll, "Symptoms of depression, acute myocardial infarction, and total mortality in a community sample," Circulation, vol. 93, no. 11, pp. 1976-1980, 1996.

[25] B. W. J. H. Penninx, A. T. F. Beekman, A. Honig et al., "Depression and cardiac mortality: results from a communitybased longitudinal study," Archives of General Psychiatry, vol. 58, no. 3, pp. 221-227, 2001.

[26] S. Zipfel, A. Schneider, B. Wild et al., "Effect of depressive symptoms on survival after heart transplantation," Psychosomatic Medicine, vol. 64, no. 5, pp. 740-747, 2002.

[27] D. E. Bush, R. C. Ziegelstein, M. Tayback et al., "Even minimal symptoms of depression increase mortality risk after acute myocardial infarction," The American Journal of Cardiology, vol. 88, no. 4, pp. 337-341, 2001.

[28] O. E. Havik, B. Sivertsen, A. Relbo et al., "Depressive symptoms and all-cause mortality after heart transplantation," Transplantation, vol. 84, no. 1, pp. 97-103, 2007.

[29] R. M. Carney, K. E. Freedland, S. A. Eisen, M. W. Rich, and A. S. Jaffe, "Major depression and medication adherence in elderly patients with coronary artery disease," Health Psychology, vol. 14, no. 1, pp. 88-90, 1995.

[30] M. R. DiMatteo, H. S. Lepper, and T. W. Croghan, "Depression is a risk factor for noncompliance with medical treatment metaanalysis of the effects of anxiety and depression on patient adherence," Archives of Internal Medicine, vol. 160, no. 14, pp. 2101-2107, 2000.

[31] D. Cukor, D. S. Rosenthal, R. M. Jindal, C. D. Brown, and P. L. Kimmel, "Depression is an important contributor to low medication adherence in hemodialyzed patients and transplant recipients," Kidney International, vol. 75, no. 11, pp. 1223-1229, 2009.

[32] A. Gehi, D. Haas, S. Pipkin, and M. A. Whooley, "Depression and medication adherence in outpatients with coronary heart disease: findings from the heart and soul study," Archives of Internal Medicine, vol. 165, no. 21, pp. 2508-2513, 2005.

[33] M. A. Dew, L. H. Roth, H. C. Schulberg et al., "Prevalence and predictors of depression and anxiety-related disorders during the year after heart transplantation," General Hospital Psychiatry, vol. 18, pp. 48S-61S, 1996.

[34] F. Dobbels, J. Vanhaecke, L. Dupont et al., "Pretransplant predictors of posttransplant adherence and clinical outcome: an evidence base for pretransplant psychosocial screening," Transplantation, vol. 87, no. 10, pp. 1497-1504, 2009.

[35] J. E. Owen, C. L. Bonds, and D. K. Wellisch, "Psychiatric evaluations of heart transplant candidates: Predicting post-transplant hospitalizations, rejection episodes, and survival," Psychosomatics, vol. 47, no. 3, pp. 213-222, 2006.
[36] S. S. Rogal, D. Landsittel, O. Surman, R. T. Chung, and A. Rutherford, "Pretransplant depression, antidepressant use, and outcomes of orthotopic liver transplantation," Liver Transplantation, vol. 17, no. 3, pp. 251-260, 2011.

[37] L. Eisenberg, "Treating depression and anxiety in primary care: closing the gap between knowledge and practice," The New England Journal of Medicine, vol. 326, no. 16, pp. 1080-1084, 1992.

[38] S. Moussavi, S. Chatterji, E. Verdes, A. Tandon, V. Patel, and B. Ustun, "Depression, chronic diseases, and decrements in health: results from the World Health Surveys," The Lancet, vol. 370, no. 9590, pp. 851-858, 2007.

[39] L. F. Berkman, J. Blumenthal, M. Burg et al., "Effects of treating depression and low perceived social support on clinical events after myocardial infarction: the Enhancing Recovery in Coronary Heart Disease Patients (ENRICHD) randomized trial," The Journal of the American Medical Association, vol. 289, no. 23, pp. 3106-3116, 2003.

[40] M. R. Costanzo, A. Dipchand, R. Starling et al., "The international society of heart and lung transplantation guidelines for the care of heart transplant recipients," Journal of Heart and Lung Transplantation, vol. 29, no. 8, pp. 914-956, 2010.

[41] P. Fusar-Poli, M. Picchioni, V. Martinelli et al., "Anti-depressive Therapies After Heart Transplantation," Journal of Heart and Lung Transplantation, vol. 25, no. 7, pp. 785-793, 2006.

[42] K. Kroenke, R. L. Spitzer, and J. B. W. Williams, "The PHQ9: validity of a brief depression severity measure," Journal of General Internal Medicine, vol. 16, no. 9, pp. 606-613, 2001.

[43] L. S. Williams, E. J. Brizendine, L. Plue et al., "Performance of the PHQ-9 as a screening tool for depression after stroke," Stroke, vol. 36, no. 3, pp. 635-638, 2005.

[44] B. Löwe, I. Schenkel, C. Carney-Doebbeling, and C. Göbel, "Responsiveness of the PHQ-9 to psychopharmacological depression treatment," Psychosomatics, vol. 47, no. 1, pp. 62-67, 2006.

[45] A. Pinto-Meza, A. Serrano-Blanco, M. T. Peñarrubia, E. Blanco, and J. M. Haro, "Assessing depression in primary care with the PHQ-9: can it be carried out over the telephone?" Journal of General Internal Medicine, vol. 20, no. 8, pp. 738-742, 2005.

[46] P. L. DiGiorgi, M. S. Reel, B. Thornton, E. Burton, Y. Naka, and M. C. Oz, "Heart transplant and left ventricular assist device costs," Journal of Heart and Lung Transplantation, vol. 24, no. 2, pp. 200-204, 2005.

[47] T. I. Shireman, J. E. Martin, and J. F. Whiting, "The cost of transplant graft maintenance following solid organ transplantation," Transplantation Proceedings, vol. 33, no. 1-2, pp. 1920-1921, 2001.

[48] M. E. Lacruz, R. T. Emeny, S. Haefner et al., "Relation between depressed mood, somatic comorbidities and health service utilisation in older adults: results from the KORA-Age study," Age and Ageing, vol. 41, no. 2, pp. 183-190, 2012.

[49] V. Myers, Y. Gerber, Y. Benyamini, U. Goldbourt, and Y. Drory, "Post-myocardial infarction depression: increased hospital admissions and reduced adoption of secondary prevention measures-a longitudinal study," Journal of Psychosomatic Research, vol. 72, no. 1, pp. 5-10, 2012.

[50] R. L. Reese, K. E. Freedland, B. C. Steinmeyer, M. W. Rich, J. W. Rackley, and R. M. Carney, "Depression and rehospitalization following acute myocardial infarction," Circulation: Cardiovascular Quality and Outcomes, vol. 4, no. 6, pp. 626-633, 2011.

[51] G. Bruschi, T. Colombo, F. Oliva et al., "Heart transplantation: 25 years' single-centre experience," Journal of Cardiovascular Medicine, vol. 14, no. 9, pp. 637-647, 2013. 


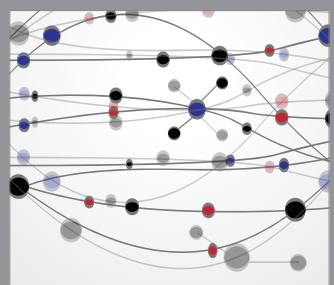

The Scientific World Journal
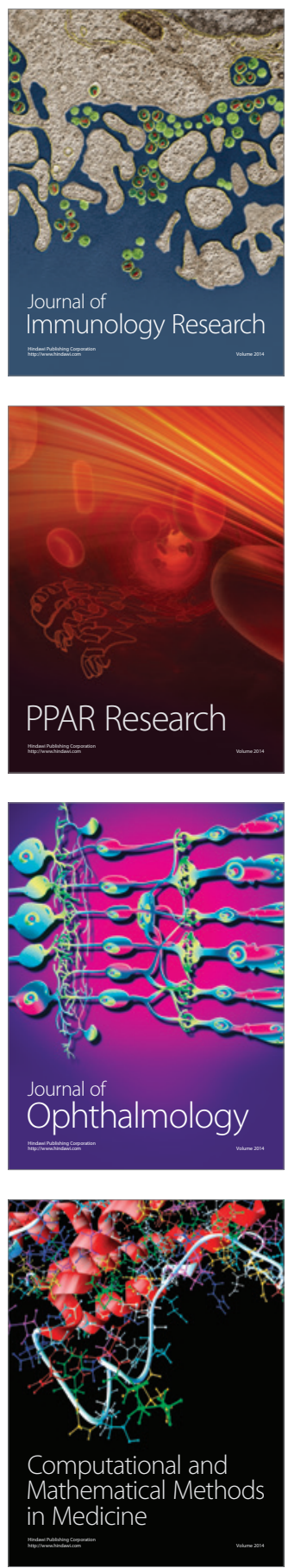

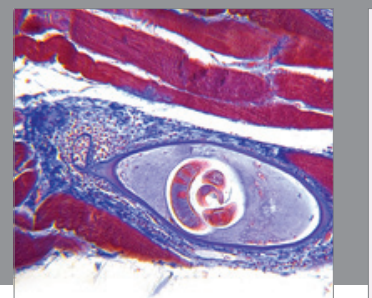

Gastroenterology

Research and Practice
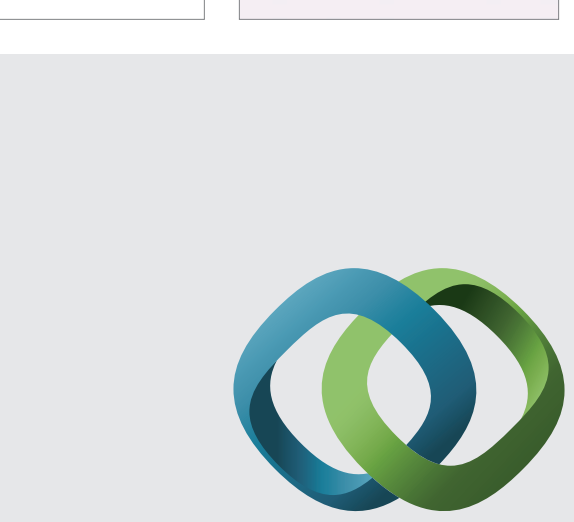

\section{Hindawi}

Submit your manuscripts at

http://www.hindawi.com

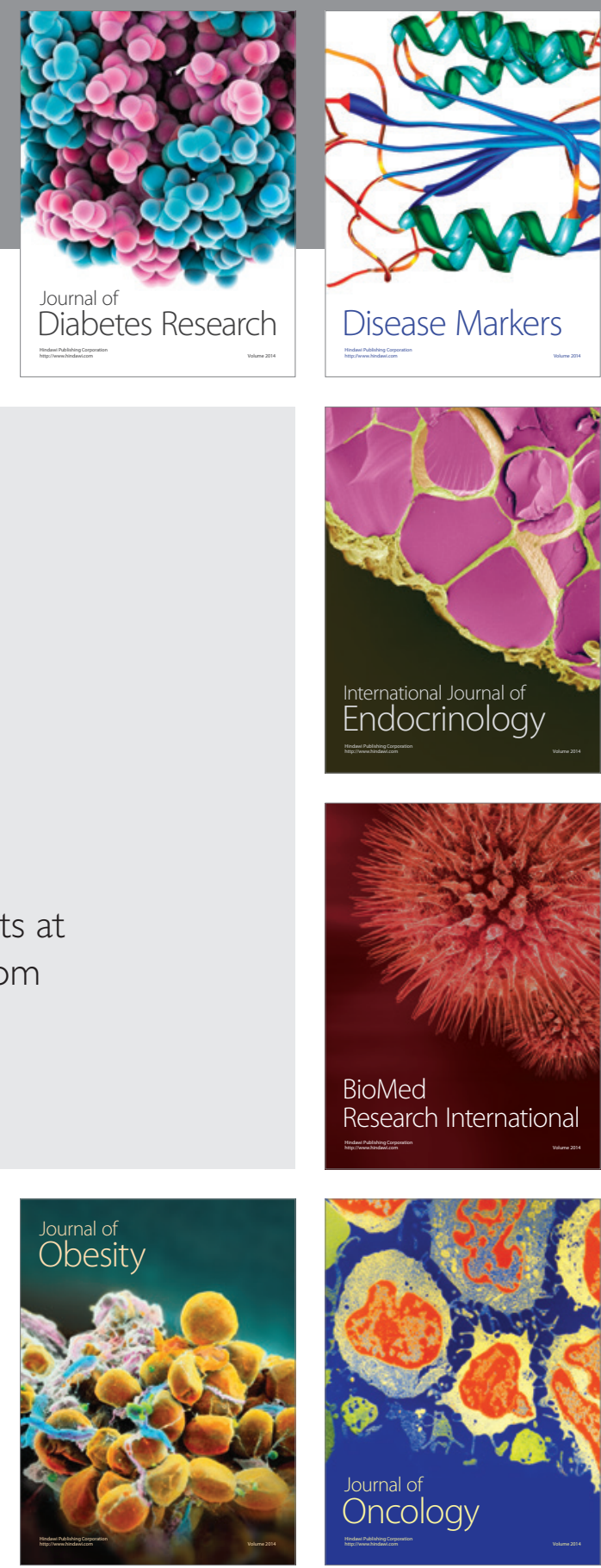

Disease Markers
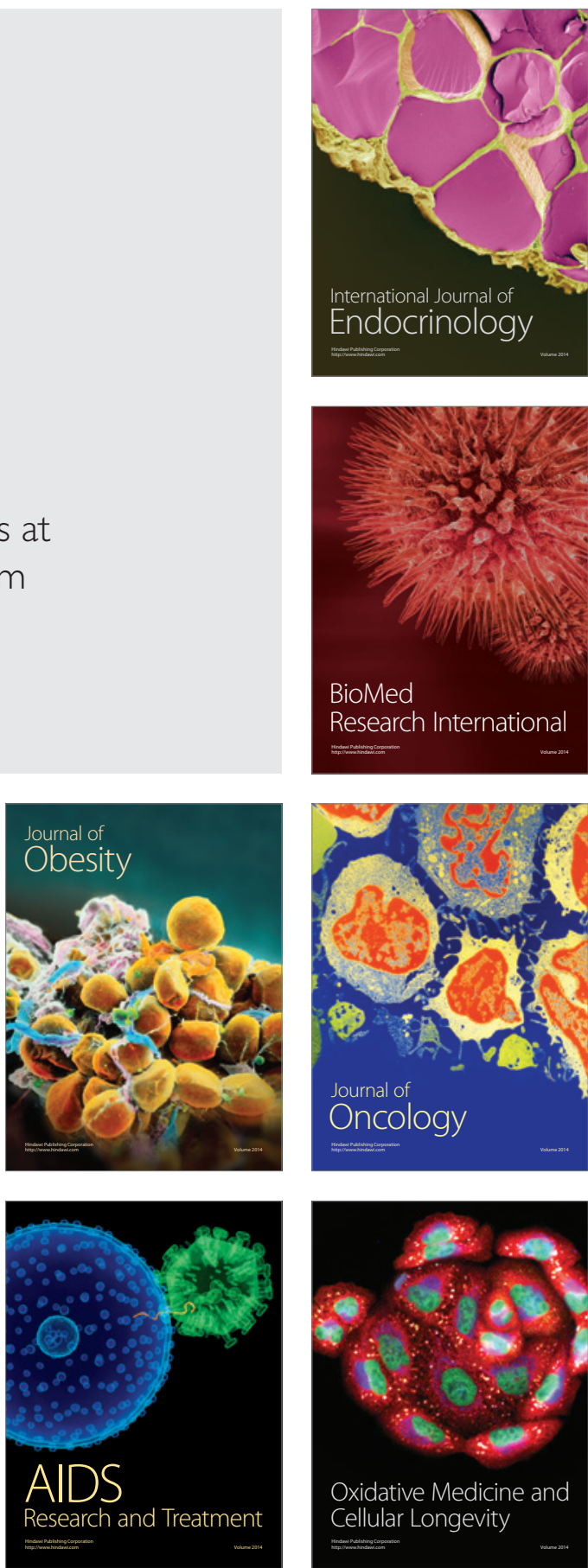